\title{
Dynamic protein adsorption in microchannels by "stop-flow" and continuous flow $\dagger$
}

\author{
Received 24th April 2005, Accepted 4th July 2005 \\ First published as an Advance Article on the web 11th August 2005 \\ DOI: $10.1039 / \mathrm{b506009e}$
}

Andrea Lionello, Jacques Josserand, Henrik Jensen and Hubert H. Girault*

This work addresses two ways of loading proteins on microchannel surfaces for immunoassay applications: the "stop-flow" and the continuous flow processes. The "stop-flow" method consists of successive static incubation periods where the bulk solution depletes upon the adsorption process. In the present paper, a multi-step "stop-flow" protein coating is studied and compared to a coating under continuous flow conditions. For the "stop-flow", a non-dimensional parameter is here introduced, indicating the adsorbing capacity of the system, by which it is possible to calculate the number of loads necessary to reach the optimum coverage. For the continuous flow, the effects on the adsorption of the kinetic rates, flow velocity and wall capacity have been considered. This study shows the importance of a careful choice of the fluid velocity to minimise the sample waste. For diffusion controlled and kinetics controlled processes, two flow velocity criteria are provided in order to obtain the best possible coverage, with the same amount of sample as with the "stop-flow".

\section{Introduction}

In previous work ${ }^{1}$ we showed that during a microimmunoassay the solution of adsorbing species (either the primary antibody or the antigen) might undergo a depletion due to the large surface-to-volume ratio intrinsic to microsystems. The depletion leads to a lower adsorption level of the species on the walls of the microsystem (compared to a bigger system, where semi-infinite diffusion occurs). This may lead to a low signal during detection. Renewing the solution by sequential fillings of the microsystem (multiple "stop-flow" incubations) or by continuously flowing the solution in the microchannel can alleviate this drawback.

The "stop-flow" method consists in stopping the flow to allow the analyte more time to diffuse to the active wall. This "stop-flow" methodology is here simulated in a multi-step way and the adsorption obtained is compared with the one obtained with flowing conditions.

Many examples of flowing heterogeneous microimmunoassays can be found where the analyte is flowed past the antibody bound to a polymer surface ${ }^{2-5}$ or functionalised surfaces. ${ }^{6}$ Sometimes, even the binding of the primary antibody is performed under flow conditions. ${ }^{7}$

The process of adsorption under flowing conditions is also encountered in some biosensors for the determination of the

Laboratoire d'Electrochimie Physique et Analytique, Institut des

Sciences et Ingénierie Chimiques (ISIC), Ecole Polytechnique Fédérale de Lausanne, CH-1015, Lausanne, Switzerland.

E-mail: hubert.girault@epfl.ch; Fax: +41 2169336 67;

Tel: +41216933151

$\uparrow$ Electronic supplementary information (ESI) available: general forms of the equations of the model (Galerkin formulation); numerical technique; effects of the velocity profile, diffusion coefficient and channel length on the adsorption; time comparison between continuous flow and "stop-flow" in a diffusion limited case. See http:// dx.doi.org/10.1039/b506009e affinity and dissociation kinetic constants of biological systems. In the Biacore biosensors, kinetic constants are usually obtained with the software accompanying the sensor. ${ }^{8}$ Some deviations from the experimental results were observed though, leading to the elaboration of models that take into account the different aspects affecting the binding. A model to interpret the interaction between any number of soluble and immobilised species on a surface was proposed ${ }^{9}$ in which special attention was brought to the rate constants of association and dissociation as the main parameters governing diffusion or reaction controlled processes. Another thorough study of the relationships between the kinetic constants and the limitation processes was done by Yarmush et al. ${ }^{10}$ Myszka et $a{ }^{11}{ }^{11}$ introduced the two compartments model in which the analyte diffuses and binds to the ligand in an unstirred layer adjacent to the surface. This model was improved ${ }^{12}$ and used several times ${ }^{13,14}$ to analyse biochemical binding processes. Another model has also been proposed for coupling transport phenomena in a flow channel with hindered diffusion transport and reaction in the hydrogel layer of a Biacore sensor. ${ }^{15}$ Also, with this device, the antibody/antigens systems were studied using a Langmuir isotherm model. ${ }^{16}$

More generally, a mathematical approach to adsorption kinetics in a flow cell has been developed by Filippov and Filippova $^{17}$ and mathematical models of adsorption and transport processes in capillary electrochromatography have been presented. ${ }^{18}$

In the present work, the finite element method (FEM) is used to study the coupling of adsorption kinetics to convection-diffusion phenomena in microchannels, providing the time evolution of the concentration of one species, both in solution and on the walls of the microchannel. It can be used to study the adsorption of the primary antibody onto a substrate or to study the reaction between a biomolecule and the attached antibody. An analytical study to provide the 
number of "stop-flow" steps necessary to obtain full coverage is addressed and validated with simulation results.

The continuous flow is studied with the FEM model and the conditions to obtain a uniform coating along the channel are analysed. This leads to criteria for setting the flow velocities that allow a comparison with the "stop-flow" procedure in terms of sample consumption. The channel length used in the simulations is $1 \mathrm{~mm}$ long, and the flow velocity range is $10-100 \mu \mathrm{m} \mathrm{s}^{-1}$. The possibility to extend the present results to longer channels by using higher velocity values (scaling based on the residence time conservation) has been experimentally verified.

\section{Theory}

\section{Adsorption in microchannels}

We consider the adsorption process according to the Langmuir isotherm model. The expression relating the concentration of analyte adsorbed on the surface, $\Gamma$, to the one in solution $C$, at equilibrium, is:

$$
\frac{\Gamma_{\mathrm{eq}}^{\text {theor }}}{\Gamma_{\max }}=\frac{K C^{\circ}}{1+K C^{\circ}}=\frac{\psi}{1+\psi}
$$

where $\Gamma_{\mathrm{eq}}{ }^{\text {theor }}$ is the surface concentration at equilibrium, $\Gamma_{\max }$ is the initial concentration of the active sites, $K$ is the thermodynamic constant of adsorption, and $C^{\circ}$ the initial concentration of the solution. The parameter $\psi$, "the motivating force to adsorption", is equal to $K C^{\circ}{ }^{19}$ In a microsystem submitted to static adsorption without renewing the solution, the analyte concentration at equilibrium is no longer $C^{\circ}$ but $C_{\text {eq }}$. As a consequence, the maximum surface concentration value attainable is $\Gamma_{\text {eq }}{ }^{\mu s y s t}$, lower than $\Gamma_{\text {eq }}{ }^{\text {theor }}$, and eqn (1) should be written as:

$$
\frac{\Gamma_{\mathrm{eq}}^{\mu s y s t}}{\Gamma_{\max }}=\frac{K C_{\mathrm{eq}}}{1+K C_{\mathrm{eq}}}
$$

\section{The "stop-flow" procedure (far from full coverage)}

This procedure consists of renewing the solute by a fast injection that is then stopped to allow an adsorption step under static conditions. As soon as the "intermediate" equilibrium $\Gamma_{\text {eq }}{ }^{\mu s y s t}$ has been attained, the depleted solution is renewed again and again up to $\Gamma_{\text {eq }}{ }^{\text {theor }}$. The number of loads needed to attain $\Gamma_{\text {eq }}{ }^{\text {theor }}$ can be estimated in the case $\Gamma_{\text {eq }}{ }^{\mu s y s t} \ll \Gamma_{\max }$, i.e. when $K C_{\text {eq }} \ll 1$. Under this assumption, $K C_{\text {eq }}$ can be neglected in the denominator of eqn (2), which becomes:

$$
\Gamma_{\text {eq }}^{\mu \text { syst }}=K C_{\text {eq }} \Gamma_{\max }
$$

One step adsorption. The total number of analyte moles $n_{\text {TOT }}$ present in the channel is equal to the number of moles injected, which distributes between the adsorbing wall and the depleted bulk: $n_{\mathrm{TOT}}=n_{\text {inj }}=n_{\text {wall }}{ }^{\text {eq }}+n_{\text {sol }}^{\text {eq }}$, where $n_{\text {inj }}\left(=C^{\circ} \mathrm{Vol}\right)$ indicates the number of moles injected into the system, $n_{\text {wall }}{ }^{\text {eq }}$ and $n_{\text {sol }}$ eq respectively the moles adsorbed on the active surface and those still in solution at equilibrium.

The mass balance can be rewritten as $n_{\mathrm{TOT}}=\Gamma_{\text {eq }}{ }^{\mu \text { syst }} A+$ $C_{\text {eq }} \mathrm{Vol}$, where $A$ and $\mathrm{Vol}$ are the active surface area and the volume of the microchannel. Keeping into account eqn (3), the following equation is obtained:

$$
\frac{n_{\mathrm{wall}}^{\text {eq }}}{n_{\mathrm{TOT}}}=\frac{K \Gamma_{\max } A V_{o l}^{-1}}{1+K \Gamma_{\max } A V_{0 l}^{-1}}=\frac{1}{1+\varphi}=\alpha
$$

where $\alpha$ indicates the relative adsorbing capacity of the system, giving the ratio between the number of moles of adsorbed analyte and the total number of moles of analyte present in the system. The parameter $\varphi$ is the dimensionless parameter introduced in ref. 1 that represents the intrinsic binding characteristic of the microchannel $\left(\varphi=V o l / A K \Gamma_{\max }\right)$. More precisely, this parameter $\varphi$ describes the number of moles adsorbed in a microsystem compared to an ideal system (in semi-infinite diffusion) with the same $\Gamma_{\max }$ and $K$. A low $\varphi$ value means that $\Gamma_{\max }$ and $K$ are high compared to the volume-to-surface ratio, leading to a consequent important bulk depletion effect.

Multi-step "stop-flow". Contrary to the first step, the initial surface concentration on the wall prior to another injection is not zero and at load $N$, a number $n_{\text {wall, }-1}{ }^{\text {eq }}$ of molecules has already been adsorbed, leading to:

$$
n_{\mathrm{TOT}, N}=n_{\mathrm{inj}}+n_{\mathrm{wall}, N-1}^{\mathrm{eq}}
$$

The number of moles injected into the channel $n_{\text {inj }}$ is considered to be the same at each step (i.e. $\left.C^{\circ} V o l\right)$. Due to the fact that the equilibrium conditions are independent of the initial distribution of the species between the surface and the solution, eqn (4) can be extrapolated to the step $N$ giving eqn (6a); substituting eqn (5) into eqn (6a) gets to eqn (6b); by analogy with eqn (6b), we can write $n_{\text {wall }, N-1}{ }^{\text {eq }}=\alpha\left(n_{\text {inj }}+n_{\text {wall }, N-2}{ }^{\text {eq }}\right)$ that can be substituted into eqn (6b) giving eqn (6c).

$$
\begin{gathered}
\frac{n_{\mathrm{wall}, N}{ }^{\mathrm{eq}}}{n_{\mathrm{TOT}, N}}=\alpha \\
n_{\mathrm{wall}, N}{ }^{\mathrm{eq}}=\alpha\left(n_{\mathrm{inj}}+n_{\mathrm{wall}, N-1}{ }^{\mathrm{eq}}\right) \\
n_{\mathrm{wall}, N}{ }^{\mathrm{eq}}=\alpha n_{\mathrm{inj}}+\alpha\left(\alpha\left(n_{\mathrm{inj}}+n_{\mathrm{wall}, N-2}{ }^{\mathrm{eq}}\right)\right)
\end{gathered}
$$

Iterating $N$ times this passage we obtain $n_{\text {wall, } N}{ }^{\text {eq }}=\left(\alpha+\alpha^{2}+\right.$ $\left.\alpha^{3}+\ldots+\alpha^{N}\right) n_{\text {inj. }}$. The geometric series $\sum_{i=0}^{n-1} a x^{i}=a\left(\frac{1-x^{n}}{1-x}\right)$ can be written in the present case, giving eqn (7):

$$
\frac{n_{\mathrm{wall}, N}^{\mathrm{eq}}}{n_{\mathrm{inj}}}=\sum_{i=0}^{N} \alpha^{i}-1=\left(\frac{1-\alpha^{N+1}}{1-\alpha}-1\right)=\frac{\alpha-\alpha^{N+1}}{(1-\alpha)}
$$

\section{Continuous flow}

When the channel (represented in Fig. 1) is submitted to transient convection-diffusion conditions, the local form of the flux conservation of the bulk concentration $C$ is given by eqn (8). The boundary condition at the active wall is expressed by eqn (9), linking the analyte consumption flux at the active wall $^{1}$ to the time evolution of its adsorbed form:

$$
\begin{gathered}
\frac{\partial C}{\partial t}+\nabla \cdot(-D \nabla C+V C)=0 \\
\frac{\partial \Gamma}{\partial t}=D\left(\frac{\partial C}{\partial y}\right)_{y=0}=k_{\mathrm{on}} C\left(\Gamma_{\max }-\Gamma\right)-k_{\mathrm{off}} \Gamma
\end{gathered}
$$




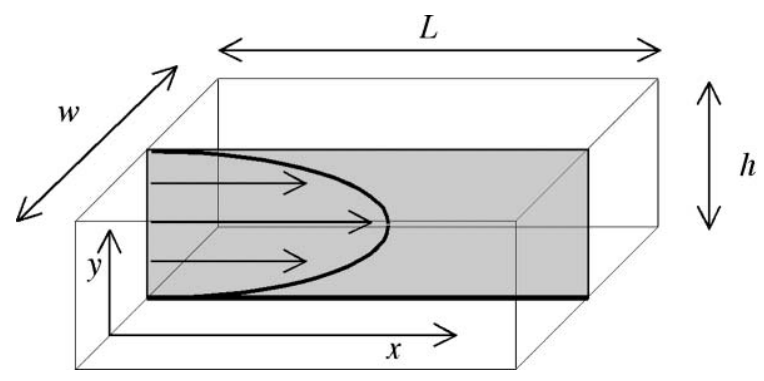

Fig. 1 Scheme of the PET microchannel (not to scale): $L=4 \mathrm{~cm}$ (the detection point is fixed at $1.5 \mathrm{~cm}$ from the outlet), $w=200 \mu \mathrm{m}$ and $h=50 \mu \mathrm{m}$. The channel is etched by laser ablation in a $100 \mu \mathrm{m}$ high PET sheet. The shaded area inside the channel represents the $2 \mathrm{D}$ area used in the simulations (in this case $L=1 \mathrm{~mm}$ ), where the PDF flow velocity is used (further information can be found in the supplementary information $\dagger$ ). The adsorbing wall is on the bottom.

where $D$ is the analyte diffusion coefficient, $k_{\text {on }}$ and $k_{\text {off }}$ are the forward and reverse rates of adsorption (the thermodynamic constant being $K=k_{\mathrm{on}} / k_{\mathrm{off}}$ ) and $\boldsymbol{V}$ is the fluid velocity vector for the pressure driven flow (PDF). For simplicity the nabla operator $\nabla$ is used: $\nabla C=\operatorname{grad} C ; \nabla \cdot$ vector $=$ divergence (vector). It is worth noting that the same kind of equations (but without convection for eqn (8), i.e. Fick's law) is used for the simulation of the "stop-flow" process.

\section{Numerical model and assumptions}

Electronic Supplementary information (ESI) is provided $\dagger$ where eqns (8) and (9) are formulated using the Galerkin method. The finite element formulation is implemented in the software Flux-Expert (iil) (Astek Rhône-Alpes, France), ${ }^{20}$ which is performed on a Silicon Graphics Octane 2 Unix workstation. The features of the model are fully described in the ESI.

The following conditions are assumed. (i) The solutions are sufficiently diluted so that the viscosity and the density of the fluid (assumed to be uniform) are not modified by concentration variations. (ii) The channel walls are assumed to be smooth and the wall capillary forces are neglected in the eventual case of a liquid/air interface. (iii) The width $w$ of the channel is much larger than its height $h$ so that the velocity gradient in the third dimension can be neglected (2D Cartesian assumption). (iv) For the multiple "stop-flow" process, the flowing load of the solution is assumed to be instantaneous. (v) Dead volume effects are neglected.

\section{Experimental}

The poly(ethylene terephthalate) (PET) channel fabrication method by laser ablation and the characteristics of the confocal microscope are described in ref. 21 and 1 respectively. The chip is inserted in a Plexiglass holder allowing the infusion of the analyte solution through the inlet of the microchannel at a constant flow rate. The device is coupled to 2 syringe pumps (Kd Scientific; accuracy of the flow rate: $\pm 1 \%$ ) via PET microtubes (incubated for 2 hours in a $\%$ BSA (SIGMA) in $0.01 \mathrm{M}$ phosphate buffer solution (PBS) to avoid IgG adsorption) connected to the Plexiglass holder with a $\mathrm{T}$ junction. A $10^{-8} \mathrm{M}$ solution of labelled antibody (Fluorolink Cy5 labelled antirabbit IgG, Amersham Pharmacia Biotech) in $0.01 \mathrm{M}$ PBS was pumped in a $4 \mathrm{~cm}$ long microchannel $(50 \times 200 \mu \mathrm{m}$ in cross section, as shown in Fig. 1) at a rate of $90 \mu \mathrm{L} \mathrm{h}^{-1}$, corresponding to $\overline{\boldsymbol{V}}=2.5 \mathrm{~mm} \mathrm{~s}^{-1}$ leading to a residence time of $10 \mathrm{~s}$, as the experimental channel length is $25 \mathrm{~mm}$ (see Fig. SI3 in the ESI $\dagger$ ).

The protein solution was pumped for a time $t_{0}$ during which adsorption occurs. After this, the washing buffer $(0.1 \%$ Tween-20 (SIGMA) in PBS) was pumped at the same inlet at a rate of $270 \mu \mathrm{L} \mathrm{h}^{-1}$ for 1 minute to ensure an efficient washing of the unadsorbed proteins. ${ }^{21}$ At this point, the fluorescence of adsorbed antibody on the channel wall was measured with the confocal microscope, giving the $t_{0}$ value. The protein solution was then pumped in the same channel for an additional time $t_{1}$, followed by washing and measurement, giving the $t_{0}+t_{1}$ value, and so forth. No appreciable delay in stopping the flow was observed while switching from the infusion of the proteins to the washing buffer. The detection spot was fixed at $2.5 \mathrm{~cm}$ from the channel inlet. The mean value and its standard deviation were taken from 5 measurements and the minimum and maximum values from the measurements are used to bound the average values in Fig. 6 .

The quantity of adsorbed antibody can be calculated from the fluorescence measurements with a calibration curve as done in ref. 1. However, to compare experiments and simulations, the fluorescence was normalised by the maximum value obtained at equilibrium. The linearisation of the Langmuir isotherm of adsorption ${ }^{1}$ of this system led to the $\Gamma_{\max }$ value for the IgG adsorption on the (PET) microchannel. As this value is largely lower than the one theoretically obtainable, multilayer adsorption is not accounted for.

\section{Results and discussion}

\section{Stop-flow process}

The "stop-flow" procedure is basically a sequence of adsorption equilibria alternated with a stepwise renewing of the bulk solution. It is illustrated by numerical simulations in Fig. 2, showing how the duration and the number of the sequential loadings required to reach $\Gamma_{\text {eq }}{ }^{\text {theor }}$ decreases with increasing $\psi$. When $\psi$ (i.e. $K C^{\circ}$ ) is multiplied by two orders of magnitude, the number of loads $N$ to reach $99 \%$ of $\Gamma_{\text {eq }}^{\text {theor }}$ is divided by 3 (from 3 to 1 ) and the time is divided by a factor of 30 .

For a low coverage situation $\left(\psi=10^{-2}\right)$, the analytical solution (eqn (7)) is represented in Fig. 3 (lines) as a function of $N$ and compared to the simulation results (markers) at different $\alpha$ values. Any microsystem is characterised by an $\alpha$ value, which can be calculated by the second term of eqn (4), and which is inversely proportional to $1+\varphi$. The parameter $\alpha$ can be used to predict how many loads are necessary to reach $\Gamma_{\text {eq }}{ }^{\text {theor }}$ (i.e. to reach $93 \%$ of the plateaus of the plots in Fig. 3, for instance 3, 7, 20 loads are necessary respectively for $\alpha=0.4$, $0.6,0.8$, the last not shown). The number of loads needed increases with $\alpha$, (i.e. with $\Gamma_{\max }, K$, and the surface-to-volume ratio $A / \mathrm{Vol}$ of the microsystem). When $\alpha \rightarrow 1$ (i.e. the wall has a great adsorbing capacity), a great number of loads are needed: this is due to the high $\Gamma_{\max } / C^{\circ}$ ratio values resulting from the assumption of low coverages (i.e. low $\psi$ ). Meanwhile, 


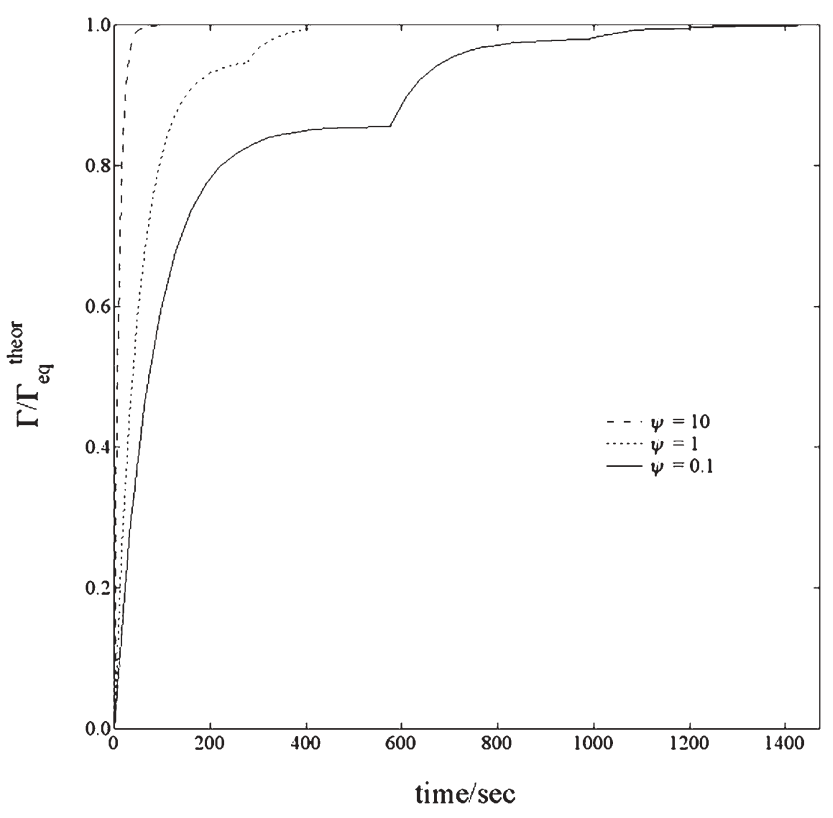

Fig. 2 Time evolution of the coverage in a $50 \mu \mathrm{m}$ high microchannel with sequential loadings of the analyte solution. The number of fillings and the time needed to attain $\Gamma_{\text {eq }}{ }^{\text {theor }}$ increases at lower concentrations. The parameters are $D=4 \times 10^{-11} \mathrm{~m}^{2} \mathrm{~s}^{-1}, \Gamma_{\max }=2 \times$ $10^{-8} \mathrm{~mol} \mathrm{~m}^{-2}, K=10^{4} \mathrm{~m}^{3} \mathrm{~mol}^{-1}\left(k_{\mathrm{on}}=100 \mathrm{~m}^{3} \mathrm{~mol}^{-1} \mathrm{~s}^{-1}, k_{\mathrm{off}}=\right.$ $0.01 \mathrm{~s}^{-1}$; kinetics controlled adsorption). $C^{\circ}=10^{-5}, 10^{-4}$, $10^{-3} \mathrm{~mol} \mathrm{~m}^{-3}$ for $\psi=0.1,1,10$ respectively. $\Gamma_{\mathrm{eq}} / \Gamma_{\max }$ theor $=9.1 \times$ $10^{-2}, 0.5$, and $9.9 \times 10^{-1}$ for $\psi=0.1,1$ and 10 respectively.

at high $\alpha$ values, the number of moles adsorbed at each load remains high for a wide range of steps, making the multiple "stop-flow" procedure effective.

\section{Continuous flow}

For the following simulations, the parameters used are shown in Table 1. The values of $K$ and $\Gamma_{\max }$ are obtained from the study of the $\mathrm{IgG}$ antibodies adsorption on PET. ${ }^{1}$ The diffusion coefficient $D$ is that of $\operatorname{IgG}\left(D=4 \times 10^{-11} \mathrm{~m}^{2} \mathrm{~s}^{-1}\right)$ and the $C^{\circ}$ value $\left(C^{\circ}=10^{-5} \mathrm{~mol} \mathrm{~m} \mathrm{~m}^{-3}\right.$, unless otherwise specified $)$ corresponds to the one used in the experiments. A PDF flow is imposed, with a value of $\overline{\boldsymbol{V}}=100 \mu \mathrm{m} \mathrm{s}^{-1} .^{10}$

Diffusion limitation. The longitudinal distribution of the adsorbed species is represented in Fig. 4 (continuous lines) for different times of simulation. A diffusion limited adsorption $\left(k_{\text {off }}=100 \mathrm{~s}^{-1}\right)^{1}$ is illustrated, which can occur when an antigen reacts with the adsorbed antibody: this reaction also can be fitted by a Langmuir isotherm, the primary antibody representing the active site for the adsorbate. ${ }^{22}$

As the proteins advance in the channel, they are instantaneously adsorbed leading to a decreasing distribution of $\Gamma$ along the wall. The entire wall is uniformly covered in $50 \mathrm{~s}$, corresponding to $5 t_{\text {res }}\left(t_{\text {res }}=L / \bar{V}=10 \mathrm{~s}\right)$. Also the volume of sample solution used $V o l_{\text {sample }}$ is equal to 5 times the volume of the channel $\mathrm{Vol}_{\text {channel, }}$, as the following normalisation can be done:

$$
\frac{t}{t_{\text {res }}}=\frac{V o l_{\text {sample }}}{V o l_{\text {channel }}}=N
$$

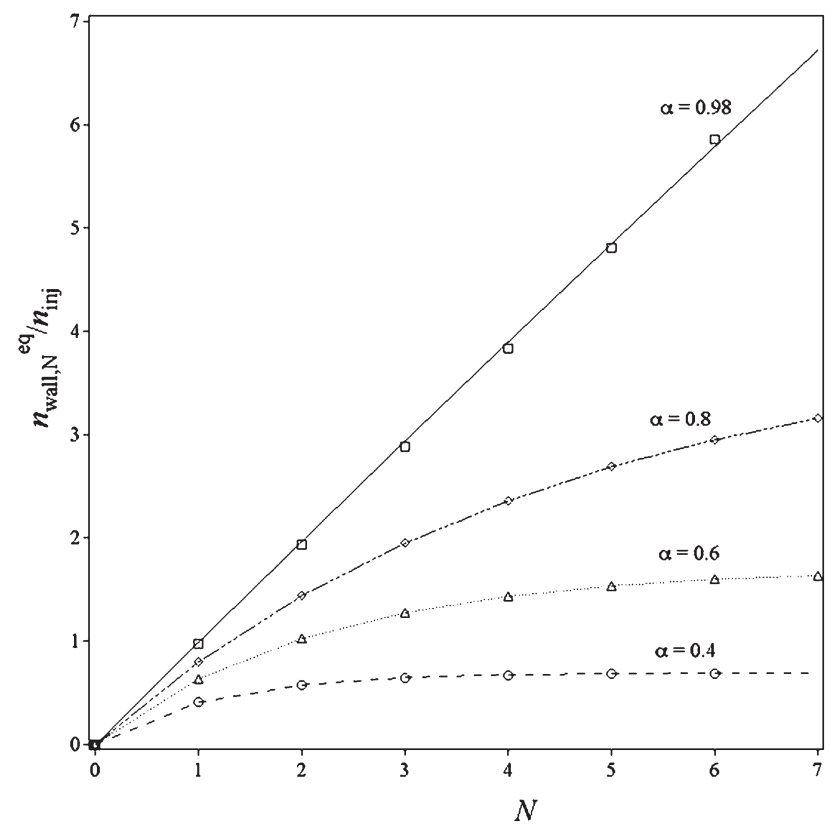

Fig. 3 Representation of the number of moles adsorbed at equilibrium compared to the number of moles injected into the microchannel as a function of the number $N$ of successive loads in a "stop-flow" procedure. The lines are calculated with eqn (7). The markers represent the results from simulations where at each step the ratio $n_{\text {wall }} \mathrm{eq} / n_{\text {inj }}$ has been evaluated: the parameters are $D=4 \times$ $10^{-11} \mathrm{~m}^{2} \mathrm{~s}^{-1}, K=2.5 \times 10^{6} \mathrm{~m}^{3} \mathrm{~mol}^{-1}\left(k_{\mathrm{on}}=2.5 \times 10^{8} \mathrm{~m}^{3} \mathrm{~mol}^{-1} \mathrm{~s}^{-1}\right.$, $k_{\text {off }}=100 \mathrm{~s}^{-1}$ : diffusion limited case), $C^{\circ}=4 \times 10^{-9} \mathrm{~mol} \mathrm{~m}^{-3}$ $(\psi=0.01) . \alpha$ was varied by changing $\Gamma_{\max }$, which is equal to $5.6 \times$ $10^{-11}, 1.4 \times 10^{-10}, 3.2 \times 10^{-10}, 3.9 \times 10^{-9} \mathrm{~mol} \mathrm{~m}^{-2}$ for $\alpha=0.4,0.6$, $0.8,0.98$ respectively.

Table 1 Parameters for the flow simulations

\begin{tabular}{ll}
\hline Parameters & Flow simulations \\
\hline$D / \mathrm{m}^{2} \mathrm{~s}^{-1}$ & $4 \times 10^{-11}$ \\
$\Gamma_{\max } / \mathrm{mol} \mathrm{m}^{-2}$ & $10^{-9}$ \\
$C^{0} / \mathrm{mol} \mathrm{m}^{-3}$ & $10^{-5}$ \\
$K / \mathrm{m}^{3} \mathrm{~mol}^{-1}$ & $10^{4}$ \\
$k_{\mathrm{on}} / \mathrm{m}^{3} \mathrm{~mol}^{-1} \mathrm{~s}^{-1}$ & $10-10^{6}$ \\
$k_{\mathrm{off}} / \mathrm{s}^{-1}$ & $10^{-3}-100$ \\
\hline
\end{tabular}

where $t$ is the injection time and $N$ corresponds to the number of channel volumes used.

To ensure the uniformity of the coverage along the channel (as after $5 t_{\text {res }}$ in Fig. 4) the injection time must be long enough to allow the solute the time to reach the end of the channel and then to diffuse to the wall. This means that the condition $t \geqslant t_{\text {res }}+t_{\text {diff }}$ must be respected, $t_{\text {diff }}$ representing the transversal diffusion time, whose order of magnitude is $h^{2} / D$. In this approach the longitudinal diffusion (along the channel) is neglected. Keeping into account the definitions of $t_{\text {res }}$ and $t_{\text {diff }}$ we finally obtain:

$$
\frac{t}{t_{\mathrm{res}}} \geq 1+\left(\frac{h}{L}\right)^{2} P e_{\mathrm{L}}
$$

where the Peclet number $P e_{\mathrm{L}}=\overline{\boldsymbol{V}} L / D$. Eqn (11) yields the minimum experimental time necessary to achieve the 


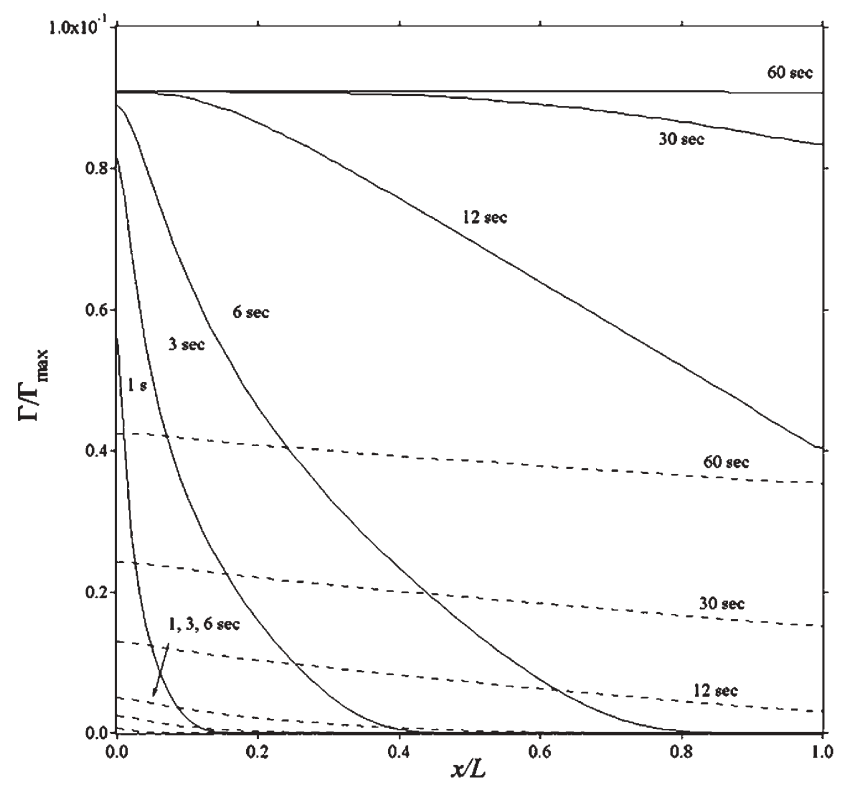

Fig. 4 Simulation results of adsorption in a microchannel, under diffusion limitation ( $k_{\text {off }}=100 \mathrm{~s}^{-1}$, continuous lines) and kinetic limitation $\left(k_{\text {off }}=10^{-2} \mathrm{~s}^{-1}\right.$, dashed lines). The plots represent $\Gamma / \Gamma_{\max }$ versus the distance from the beginning of the channel $(x)$ normalised by the channel length $(L)$ at $1,3,6,12,30$ and $60 \mathrm{~s}$ after the beginning of the injection. $D=4 \times 10^{-11} \mathrm{~m}^{2} \mathrm{~s}^{-1}, \Gamma_{\max }=10^{-9} \mathrm{~mol} \mathrm{~m} \mathrm{~m}^{-2}$, $K=10^{4} \mathrm{~m}^{3} \mathrm{~mol}^{-1}, C^{\circ}=10^{-5} \mathrm{~mol} \mathrm{~m}^{-3}, \overline{\boldsymbol{V}}=100 \mu \mathrm{m} \mathrm{s}^{-1}$. As $\psi=0.1$, $\Gamma_{\mathrm{eq}}{ }^{\text {theor }} / \Gamma_{\max }=9.1 \times 10^{-2}$.

uniformity of the coating, if the velocity of the flow is fixed by other experimental constraints.

To minimise the volume used (i.e. $N$ in eqn (10)), we must increase $t_{\text {res }}$, reducing the fluid velocity. This is shown in Fig. 5a, where the impact of different fluid velocities on the coverage uniformity along the channel is illustrated. The growth of the coverage at the beginning and at the end of the channel is reported versus the relative amount of sample used, and for the $\overline{\boldsymbol{V}}$ values $=100,30,10 \mu \mathrm{m} \mathrm{s}^{-1}$. It is illustrated how the difference between the coverages at the beginning and at the end of the channel (i.e. the non-uniformity of the coating) decreases when the velocity of the flow is reduced. As expected, the consumption of sample to reach the full coverage decreases when the velocity decreases: to obtain $99 \%$ of the inlet coverage at the channel outlet, 4.3, 2.5 and $1.9 \mathrm{Vol}_{\text {channel }}$ are respectively needed.

To choose the optimum velocity, one can fix a number $N_{\max }$ of $\mathrm{Vol}_{\text {sample }}$ that should not be exceeded. For example, if a waste not higher than that obtained with the "stop-flow" method is wanted, this number $N_{\max }$ can be fixed from Fig. 3, after calculating the coefficient $\alpha$ of the system. Introducing $N_{\max }$ in eqn (11) by the way of eqn (10), i.e. $t / t_{\text {res }}=N_{\max }$, leads to:

$$
\overline{\boldsymbol{V}} \leq\left(N_{\max }-1\right) \frac{D L}{h^{2}}
$$

that grants for $99 \%$ of uniformity along the channel with the desired waste.t We can apply this methodology to the

\# The validity of eqn (12) has been verified for systems where $P e_{\mathrm{h}}=$ $\overline{\mathrm{V}} h / D \geqslant 10$
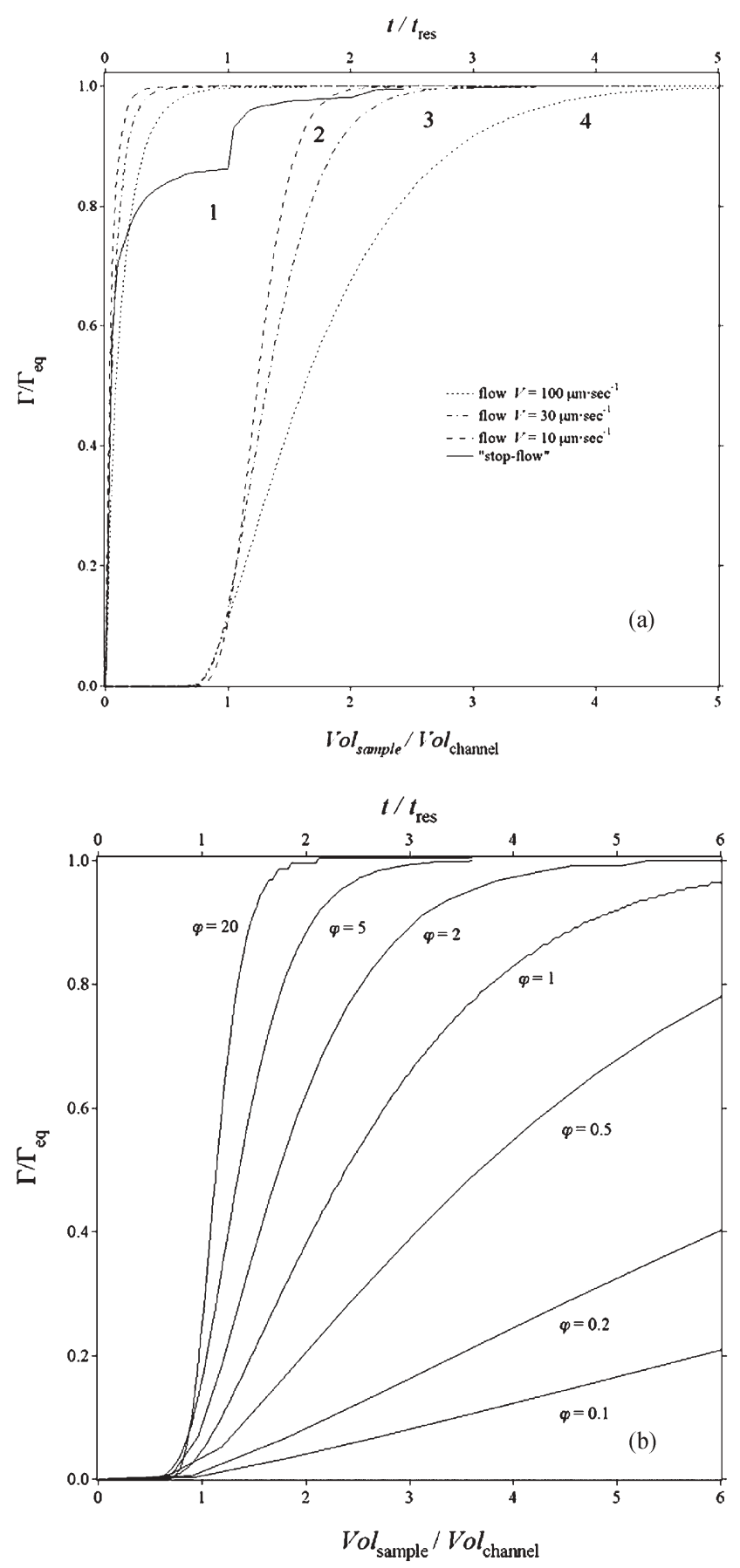

Fig. 5 (a) Evolution of the coverage at the inlet (set of plot to the left) and at the outlet (set of plots to the right) of the microchannel wall as a function of the volume of sample used, normalised by the volume of the channel $\left(V o l_{\text {sample }} / V o l_{\text {channel }}\right)$. On top axis time is normalised by the residence time $L / \overline{\boldsymbol{V}}$. The adsorption is diffusion controlled $\left(k_{\mathrm{off}}=\right.$ $100 \mathrm{~s}^{-1}$ ). Flow velocities are $\overline{\boldsymbol{V}}=100$ (short dashed lines), 30 (dashed and dotted lines), $10 \mu \mathrm{m} \mathrm{s}^{-1}$ (long dashed lines). The other parameters are those of Fig. 4. The numbers under the stop-flow plots represent the steps. (b) Evolution of the coverage at the end of the channel as a function of the residence time for different $\varphi$ values: $\Gamma_{\max }$ was changed, the other parameters are those of Fig. 5a. $\overline{\boldsymbol{V}}=30 \mu \mathrm{m} \mathrm{s}^{-1}$ so that the case $\varphi=5$ corresponds to the dashed line in Fig. 5a. 
adsorption system represented in Fig. 5a, characterised by $\alpha=0.167$. From a plot like those in Fig. 3 or eqn (7), it is possible to calculate $N_{\max }=3$ (for $99 \%$ of the $\Gamma_{\mathrm{eq}}{ }^{\text {theor }}$ ) with the "stop-flow" procedure, which is also confirmed by the full line curve of Fig. 5a. From eqn (12), for $N_{\max }=3$, we obtain $\overline{\boldsymbol{V}}=32 \mu \mathrm{m} \mathrm{s}^{-1}$ : as just seen above, this flow velocity leads to using $2.5 \mathrm{Vol}_{\text {channel }}$, respecting the desired sample consumption. More generally, choosing for instance $N_{\max }=7$ and 2 (the first not shown as it is out of scale) leads to imposing $\overline{\boldsymbol{V}}$ values $\leqslant 96$ and $16 \mu \mathrm{m} \mathrm{s}^{-1}$ (in agreement with the velocity values used in Fig. 5a, for which 4.3 , and $1.9 \mathrm{Vol}_{\text {channel }}$ are respectively used). The criterion (eqn (12)) is then confirmed as a valid rule to have less or the same waste than with the "stop-flow" for a given degree of uniformity of the adsorbed analyte along the channel.

The same comparison is illustrated in Fig. SI4 in the ESI $\dagger$ as a function of the absolute time. If time is the priority, the continuous flow becomes the fastest method when using high flow velocities, employing a great amount of sample.

It must be stated that the criterion (eqn (12)) is valid when the microsystem is characterised by a $\varphi$ value higher than 5 (as in Fig. 5a). In Fig. 5b the coating evolution as a function of time at the end of the channel is shown in systems with different $\varphi$ values and for a solution velocity $\overline{\boldsymbol{V}}=30 \mu \mathrm{m} \mathrm{s}^{-1}$. At low $\varphi$ values the adsorbing capacity of the system is very high and the residence time must be greater than the time to fill the adsorbing wall (which can be much longer than the $t_{\text {diff }}$ to cross the channel). This leads to an increased time to complete the adsorption by orders of magnitude (20 times the $t_{\text {res }}$ for $\varphi=0.1$, with $t_{\text {res }}=30 \mathrm{~s}$ ) and to a reduction of the velocity value given in eqn (12). However the $\varphi$ values range in which eqn (12) holds covers the majority of the common experimental cases (adsorption on polymers without surface modifications). ${ }^{23}$

A comparison between the number of "stop-flow" steps and the number of $t_{\text {res }}$ to be used with the continuous flow to reach full coverage (by simulations up to the equilibrium) is made in Table 2 for different $\varphi$ values. The number of "stop-flow" steps is obtained with the analytical method that was compared with simulation results in Fig. 3. The two procedures are always comparable (at $\psi=10^{-3}$ and $\overline{\boldsymbol{V}}=30 \mu \mathrm{m} \mathrm{s}^{-1}$, which respects eqn (12)). The difference with the simulations carried out with $\psi=0.1$ is due to the fact that the analytical solution presumes independence from $C^{\circ}(\psi \ll 1)$.

Table 2 Number of "stop-flow" steps needed to reach $99 \%$ of the plateau values in plots like those of Fig. 3 and number of $t_{\text {res }}$ necessary to reach $99 \%$ of the full coverage with a continuous flow $\left(\overline{\boldsymbol{V}}=30 \mu \mathrm{m} \mathrm{s}^{-1}\right)$ as a function of $\alpha$ and the corresponding $\varphi$ (parameters of Fig. 5b). The values of $t_{\text {res }}$ necessary with the flow mode are reported for $\psi=10^{-3}$ (value at which the kinetics of adsorption is independent of concentration) and for $\psi=0.1$ : for this latter value, an agreement with the calculated values is found just for $\alpha<0.5$

\begin{tabular}{lllll}
\hline$\varphi$ & $\alpha$ & $\begin{array}{l}N \text { "stop-flow" } \\
\text { analytic }\end{array}$ & $\begin{array}{l}N t_{\text {res }} \text { simul; } \\
\psi=10^{-3}\end{array}$ & $\begin{array}{l}N t_{\text {res }} \text { simul; } \\
\psi=0.1\end{array}$ \\
\hline 0.1 & 0.91 & 47 & 45 & 30 \\
0.2 & 0.83 & 25 & 29 & 14 \\
0.5 & 0.66 & 11 & 13 & 7 \\
1 & 0.5 & 6 & 6 & 5 \\
2 & 0.33 & 4 & 4 & 3 \\
5 & 0.166 & 3 & 3 & 3 \\
\hline
\end{tabular}

Kinetic limitation (see Fig. 4, dashed lines). The kinetic rates of adsorption chosen here are very low as during the antibody adsorption on the walls of a microtiter well $\left(k_{\text {off }}\right.$ values are $10^{-2} \mathrm{~s}^{-1}$, slightly faster than the kinetics of $\mathrm{IgG}$ adsorption on laser-ablated PET). ${ }^{1}$ The theoretical coverage $\Gamma_{\text {eq }}{ }^{\text {theor }}$ (i.e. $\left.9.1 \times 10^{-2} \Gamma_{\max }\right)$ is attained after a long time (100 minutes, not shown) through quasi-uniform concentration profiles (as under incubation). In fact, the time required for the adsorption $\left(t_{\text {reac }}=C^{\circ} / k_{\text {on }}=1000 \mathrm{~s}\right)^{13}$ is much greater than the difference of exposition time of the wall to the analyte between the inlet and the outlet of the channel $(x / \bar{V}=10 \mathrm{~s}$ at the channel outlet for $\overline{\boldsymbol{V}}=100 \mu \mathrm{m} \mathrm{s}^{-1}$ ). Introducing a continuous flowing process doesn't bring any improvement to this adsorption step or would require a very low fluid velocity to let the residence time be greater than the reaction time. By analogy to the case of diffusion limitation, we can derive the following criterion for the velocity:

$$
\overline{\boldsymbol{V}}<\left(N_{\max }-1\right) k_{\mathrm{on}} C^{\circ} L
$$

From this criterion, we can predict if a flow can be experimentally used or not, especially with low concentrations or slow kinetics.

In Fig. 6, a comparison between the "stop-flow" and the continuous flow under kinetic limitation is shown as a function of the absolute time, which is an important factor when the

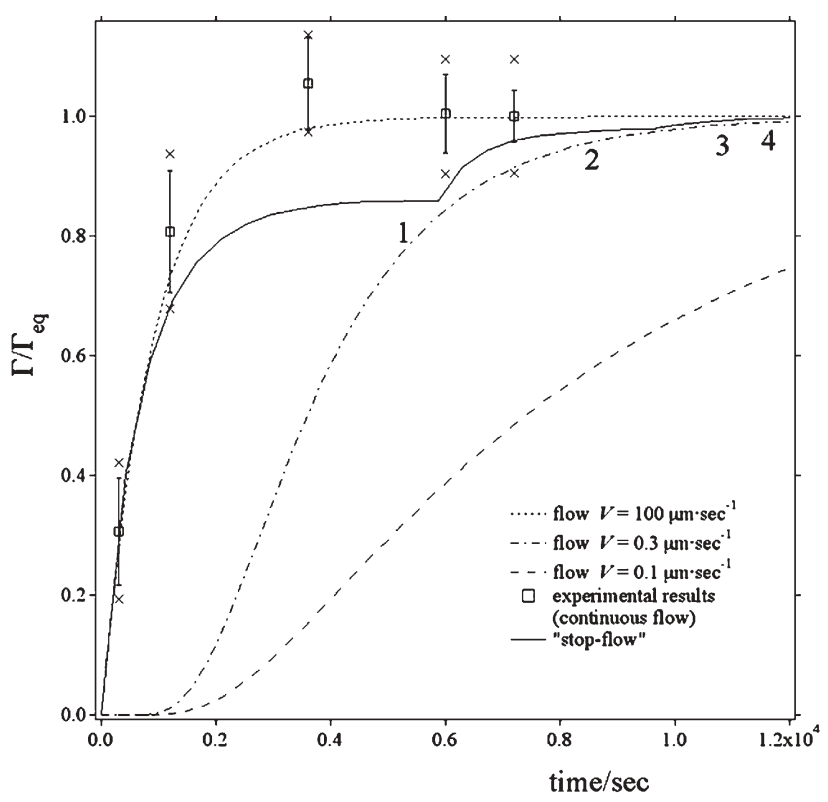

Fig. 6 Time evolution of the adsorption at the end of the channel under continuous flow and stop-flow conditions for a kinetic controlled process $\left(k_{\text {off }}=10^{-3} \mathrm{~s}^{-1}\right), \bar{V}=100 \mu \mathrm{m} \mathrm{s}^{-1}, 0.3 \mu \mathrm{m} \mathrm{s}^{-1}$ and $0.1 \mu \mathrm{m} \mathrm{s}^{-1}$. The other parameters are those of Fig. 5. The markers (open squares) show the experimental results for IgG adsorption on PET microchannels. The minimum and the maximum values of the experiments (crosses) are used to bound the average values. A $10^{-8} \mathrm{M}$ $\operatorname{IgG}$ solution in PBS was pumped into the PET channel with a pressure driven flow with a velocity of $90 \mu \mathrm{L} \mathrm{h}{ }^{-1}$ (corresponding to $\bar{V}=2.5 \mathrm{~mm} \mathrm{~s}^{-1}$ leading to the same residence time of $10 \mathrm{~s}$, as the experimental channel length is $25 \mathrm{~mm}$, as described in Fig. SI3 in the ESI $\dagger$ ). 
kinetics strongly limits the adsorption. Under "stop-flow" conditions, 4 sequential static loads are required to reach $99 \%$ of the theoretical $\Gamma_{\mathrm{eq}}{ }^{\text {theor }}$ value, and a volume of $4 \times 10^{-2} \mu \mathrm{L}$ of protein solution is needed (i.e. 4 times the channel volume). For $N_{\max }=4$, a continuous flow velocity $\overline{\boldsymbol{V}}=0.3 \mu \mathrm{m} \mathrm{s}^{-1}$ is calculated from eqn (13), and then imposed in the simulations. A time $t=1.2 \times 10^{4} \mathrm{~s}$ is needed to reach $99 \%$ of the theoretical coverage with this velocity value. This leads to use of $3.6 \times 10^{-2} \mu \mathrm{L}$, which is comparable with the one used in the "stop-flow". The criterion (eqn (13)) is then confirmed as valid to minimise the sample. The case presented here is an extreme one, as the kinetics of adsorption are very low and require flow velocity values that are difficult to achieve experimentally. On the other hand, for intermediate cases of kinetics, the criterion (eqn (13)) can be helpful to determine if the continuous flow can be used or not. $\S$

From an experimental point of view, controlling the flow in a microchannel is not always an easy task. The "stop-flow" approach alleviates the difficulty as the sample renewal can be done rapidly without controlling the flow rate, even if it implies a control of the sample volume to avoid exaggerated waste.

The experimental results of the IgG adsorption on PET under flow conditions are also shown (markers) in Fig. 6 to check the accordance with the simulations. High flow rates are important to minimize transport limitations and to allow an accurate estimation of the kinetic constants. ${ }^{15}$ Therefore a pressure driven flow rate of $90 \mu \mathrm{L} \mathrm{h}^{-1}$ was imposed (corresponding to the same residence time as for the simulation at $\overline{\boldsymbol{V}}=100 \mu \mathrm{m} \mathrm{s}^{-1}$, with the couple $(L, \overline{\boldsymbol{V}})=(25 \mathrm{~mm}$, $\left.2500 \mu \mathrm{m} \mathrm{s}^{-1}\right)$ instead of $\left(1 \mathrm{~mm}, 100 \mu \mathrm{m} \mathrm{s}^{-1}\right.$ ). (See the validation in Fig. SI3 in the ESI†.)

Good agreement is found between the simulation and the experimental results. The adsorption of $\mathrm{IgG}$ on PET is confirmed to be under kinetic control. ${ }^{1}$ The experimental coverage values are slightly greater compared to the simulated ones. This may be due to the fact that immunosorption kinetics may be enhanced by the forced convection during the filling of the channel. ${ }^{21}$

Further information on the effect of the solute diffusion coefficient and of the type of flow (pressure induced and electroosmotic flows) on the coatings can also be found in the ESI†. In particular it is verified that the results of this work can be extrapolated to longer channels, provided that the residence time is respected (e.g. the results previously obtained for the couple $(L, \bar{V})=\left(1 \mathrm{~mm}, 100 \mu \mathrm{m} \mathrm{s}^{-1}\right)$ also apply to results obtained in a system where $\left.(L, \overline{\boldsymbol{V}})=\left(1 \mathrm{~cm}, 1 \mathrm{~mm} \mathrm{~s}^{-1}\right)\right)$.

\section{Conclusions}

Due to the solution depletion occurring during static adsorption in microsystems, ${ }^{1}$ two methods to renew the solution are studied in order to obtain the best possible coverage of the active adsorbing wall: the "stop-flow" and the continuous flow processes. To do this, a finite element model has been

$\S$ The validity of eqn (13) depends on the $P e_{\mathrm{L}}$ (here $P e_{\mathrm{L}}=7.5$ ) as the typical length for the longitudinal diffusion $\delta \cong L$ (the solute diffusion length here is $\left.\delta=(\pi D t)^{1 / 2} \cong 1 \mathrm{~mm}\right)$. Consequently, for $\delta=1 \mathrm{~mm}$, $V_{\text {diff }}=D / \delta=0.04 \mu \mathrm{m} \mathrm{s}^{-1}$, confirming that it can be neglected. developed considering the transient convection diffusion of one species in solution coupled to the adsorption kinetics on the active surface.

As the multiple "stop-flow" procedure is done with sequential static loads, an analytical expression is provided by which the number of necessary loads can be predicted (in a situation far from full coverage, i.e. low concentration solutions). Finally, good agreement is found between experimental results and the simulations.

For the continuous flow process, the effects on the coating of different adsorption kinetic rates have been studied. In diffusion limited cases, uniform adsorption coverages are obtained for flow velocity values $\overline{\boldsymbol{V}}<\left(N_{\max }-1\right) D L / h^{2}$, where $N_{\text {max }}$ corresponds to the number of volumes of sample that we want to use. $N_{\max }$ can be fixed equal to the number of steps for the "stop-flow" mode, giving the velocity range for which the continuous flow is competitive with the "stop-flow" in terms of sample waste (for $99 \%$ of coating uniformity along the channel). Similarly, under kinetic control, the value $\overline{\boldsymbol{V}}=$ $\left(N_{\max }-1\right) k_{\text {on }} C^{\circ} L$ ensures the theoretical coverage using the same amount of sample as the "stop-flow". These comparisons underline the interest of the "stop-flow", especially with slow kinetics, low concentrations or short channels implying too slow velocity values for the continuous flow.

Measurement of IgG adsorption in a PET microchannel under flow conditions has been performed, showing a good agreement with the simulation results and confirming confocal microscopy as a useful and simple tool to investigate adsorption in microsystems. The results obtained with this model can be extended to longer channels, after scaling the velocity flow.

\section{Acknowledgements}

The Research Program UE 1.1-LIFE-GENERIC (Project: "MICROPROTEOMICS: New microfluidic-mass spectrometry technologies for high performance proteomics" $\mathrm{N}^{\circ}$ OFES '01.1821) is gratefully acknowledged for financial support.

\section{References}

1 A. Lionello, J. Josserand, H. Jensen and H. H. Girault, Lab Chip, 2005, 5, 254-260.

2 E. Eteshola and M. Balberg, Biomed. Microdevices, 2004, 6, 7-9.

3 N. Malmstadt, A. S. Hoffman and P. S. Stayton, Lab Chip, 2004, 4, 412-415.

4 K. Sato, M. Tokeshi, T. Odake, H. Kimura, T. Ooi, M. Nakao and T. Kitamori, Anal. Chem., 2000, 72, 1144-1147.

5 J. Yakovleva, R. Davidsson, A. Lobanova, M. Bengtsson, S. Eremin, T. Laurell and J. Emneus, Anal. Chem., 2002, 74, 2994-3004.

6 O. E. Galanina, M. Mecklenburg, N. E. Nifantiev, G. V. Pazynina and N. V. Bovin, Lab Chip, 2003, 3, 260-265.

7 A. Dodge, K. Fluri, E. Verpoorte and N. F. de Rooij, Anal. Chem., 2001, 73, 3400-3409.

8 R. Karlsson, A. Michaelsson and L. Mattsson, J. Immunol. Methods, 1991, 145, 229-240.

9 R. W. Glaser, J. Immunol. Methods, 1993, 160, 141-142.

10 M. L. Yarmush, D. B. Patankar and D. M. Yarmush, Mol. Immunol., 1996, 33, 1203-1214.

11 D. G. Myszka, T. A. Morton, M. L. Doyle and I. M. Chaiken, Biophys. Chem., 1997, 64, 127-137.

12 D. G. Myszka, X. He, M. Dembo, T. A. Morton and B. Goldstein, Biophys. J., 1998, 75, 583-594. 
13 T. Mason, A. R. Pineda, C. Wofsy and B. Goldstein, Math. Biosci., 1999, 159, 123-144.

14 C. Wofsy and B. Goldstein, Biophys. J., 2002, 82, $1743-1755$.

15 V. Sikavitsas, J. M. Nitsche and T. J. Mountziaris, Biotechnol. Prog., 2002, 18, 885-897.

16 P. Pham, PhD thesis, Institut National Polytechnique de Grenoble, Grenoble, 2001

17 L. K. Filippov and N. L. Filippova, J. Colloid Interface Sci., 1997, 189, $1-16$.
18 M. Paces, J. Kosek, M. Marek, U. Tallarek and A. SeidelMorgenstern, Electrophoresis, 2003, 24, 380-389.

19 W. H. Reinmuth, J. Phys. Chem., 1961, 65, 473.

20 Astek Rhône-Alpes, A.: 1 place du Verseau, 38130 Echirolles, France, flux-expert@astek.fr.

21 J. S. Rossier, G. Gokulrangan, H. H. Girault, S. Svojanovsky and G. S. Wilson, Langmuir, 2000, 16, 8489-8494.

22 B. J. Horstmann and H. A. Chase, Chem. Eng. Res. Des., 1989, 67, 243-254.

23 J. S. Rossier and H. H. Girault, Lab Chip, 2001, 1, 153-157. 